\title{
Parity Effect in the Ground state Localization of Antiferromagnetic Chains Coupled to a Ferromagnet
}

\author{
Simon Holzberger, ${ }^{1}$ Tobias Schuh, ${ }^{1}$ Stefan Blügel, ${ }^{2}$ Samir Lounis, ${ }^{2}$ and Wulf Wulfhekel ${ }^{1}$ \\ ${ }^{1}$ Physikalisches Institut, Karlsruhe Institute of Technology, Wolfgang-Gaede-Strasse 1, 76131 Karlsruhe, Germany \\ ${ }^{2}$ Peter Grünberg Institut and Institute for Advanced Simulation, Forschungszentrum Jülich and JARA, 52425 Jülich, Germany
} (Received 20 October 2012; revised manuscript received 13 January 2013; published 10 April 2013)

We investigate the ground states of antiferromagnetic Mn nanochains on $\mathrm{Ni}(110)$ by spin-polarized scanning tunneling microscopy in combination with theory. While the ferrimagnetic linear trimer experimentally shows the predicted collinear classical ground state, no magnetic contrast was observed for dimers and tetramers where noncollinear structures were expected based on $a b$ initio theory. This striking observation can be explained by zero-point energy motion for even-numbered chains derived within a classical equation of motion leading to nonclassical ground states. Thus, depending on the parity of the chain length, the system shows a classical or a quantum behavior.

Magnetism is ultimately caused by the spin degree of freedom of the electrons. If phase coherence of the electrons was preserved, the quantum nature of spin would potentially allow us to encode quantum information in spintronic devices [1]. Thus, realizing magnetic quantum devices necessarily involves the understanding of the spin of nanoscopic structures on a quantum mechanical level. Antiferromagnetic nanostructures are by far not as well studied as their ferromagnetic counterparts. This deficiency lies in the inherent experimental and theoretical difficulties which have to be overcome to understand antiferromagnets. To date, even for very simple structures such as the onedimensional antiferromagnetic chain, the ground state is unknown. While neutron diffraction of one-dimensional antiferromagnets often revealed a simple, i.e., classical, alternating orientations of the spins [2]—called the Néel state-half integer spin chains are, for example, expected to be in a complex entangled ground state [3]. The ground state becomes even more complex when competing exchange interactions exist, leading to magnetic frustration, noncollinear spin structures [4] or to correlated ground states predicted by the Anderson resonating valence bond model [5]. Geometric frustration of the Heisenberg antiferromagnet on a triangular lattice is the standard example of a magnetically frustrated system [6-9]. Here, we show that antiferromagnetic chains display a classical Néel state for odd-numbered length and an entangled state for evennumbered length when competing exchange interactions and spin-orbit interactions are present. Thus, the chains alternate between opposite nature of the ground states just by the removal or addition of a single atom.

Lounis et al. showed theoretically for $\mathrm{Mn}$ on $\mathrm{Ni}(100)$ that frustration arises from the antiferromagnetic coupling within the Mn chain competing with the ferromagnetic coupling of the chain atoms to the substrate. This frustration can lead to an even-odd effect, where the magnetic structure crucially depends on the parity of the number of atoms in the chain [10]. Here, a Ni(110) surface was chosen instead of a $\mathrm{Ni}(100)$ surface as it favors the formation of linear Mn chains due to its crystal symmetry, and lateral manipulation of adatoms on fcc(100) surfaces is not feasible [11] but has been realized on fcc(110) surfaces [12]. Magnetically, sole consideration of the predominant antiferromagnetic coupling within the Mn chain leads to an antiparallel order of the magnetic moments. Therefore, odd-numbered chains exhibit a net magnetic moment, in contrast to even-numbered ones. Switching on the weaker ferromagnetic coupling between the atoms of the chain and the substrate thus acts differently on the two kinds of chains. Odd-numbered ones retain their collinearity, and the net moment of the chain aligns with that of the substrate, which is in-plane due to shape anisotropy. Evennumbered chains, however, develop a more complex ground state. In a presumed collinear state, the total magnetic exchange energy of the Mn chains to the ferromagnetic substrate is independent of the direction of the $\mathrm{Mn}$ moments. It can, however, be lowered when a noncollinear spin-structure develops. While magnetic exchange energy has to be paid to tilt the spins of the even-numbered chain from the ideal collinear state, a net spin of the chain develops that points in the direction of the substrate magnetization, thus giving rise to an energy gain due to the exchange with the substrate.

Mn chains of lengths up to six atoms on a Ni(110) surface were simulated using the Korringa-Kohn-Rostoker Green function method $[13,14]$ as expressed within density functional theory (DFT) taking into account noncollinear spin structures within the chains and the substrate and relaxations $[10,15]$. The results were then mapped to a classical Heisenberg model in which magnetic exchange energies between first-neighbor atoms were taken into account [16]. As shown before for chains on $\mathrm{Ni}(001)$ [10], this model catches the important features observed in the ab initio calculations. 


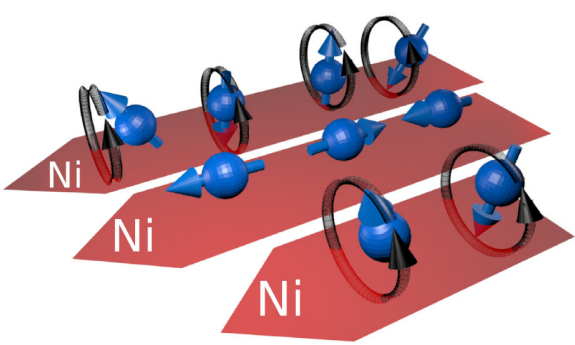

FIG. 1 (color online). DFT ground states of antiferromagnetically coupled Mn chains on $\mathrm{Ni}(110)$. While the linear trimer shows a collinear ferrimagnetic order with magnetic moments (blue vectors) parallel to the Ni magnetization (red arrow), evennumbered chains show a noncollinear magnetic structure. Without spin-orbit interaction, the magnetic moments can be coherently rotated around the Ni magnetization without changing the energy as depicted by the black circles.

Figure 1 illustrates the ground states of linear dimer, trimer, and tetramer chains on $\mathrm{Ni}(110)$ calculated within this framework. As expected, the trimer chain is in a collinear magnetic state where the net spin of the chain is aligned to the substrate magnetization. The even-numbered chains show a noncollinear magnetic configuration with moments strongly deviating from the substrate magnetization direction. Neglecting spin-orbit interaction, the magnetic moments of the chains can be rotated coherently around the direction of magnetization of the substrate without affecting the total energy [17]. When spin-orbit interaction is taken into account, the rotational degeneracy is lifted and the Mn moments prefer an out-of-plane orientation, while $\mathrm{Ni}(110)$ is magnetized in the surface plane [18]. Thus, two degenerate ground states are predicted.

So far there has been no experimental report on this effect. Currently, only spin-polarized scanning tunneling microscopy (Sp-STM) can reveal antiferromagnetism on the atomic scale [19,20]. Low-temperature STM has been used to investigate the quantum nature of small magnetic clusters [21-26]. Furthermore, STM is capable of moving adatoms, thus offering the possibility of assembling and probing at the same time [12]. In this work a home-built STM operating at $4.2 \mathrm{~K}$ and under ultrahigh vacuum conditions was used in combination with $W$-tips coated with 10 monolayers (ML) of Fe, $15 \mathrm{ML}$ of $\mathrm{Mn}$, or $30 \mathrm{ML}$ of Co for the spin-polarized measurements.

We first deposited 0.02 ML Mn with the sample held at $4.2 \mathrm{~K}$, showing primarily single $\mathrm{Mn}$ adatoms [see Fig. 2(a)]. Mn chains with the intrinsic nearest-neighbor spacing of $2.49 \AA$ were then assembled by atomic manipulation along the close-packed $\langle 1 \overline{1} 0\rangle$ direction of the substrate [see Fig. 2(b)]. The assembly was limited to tetramers, as longer chains were unstable due to the large lattice mismatch between $\mathrm{Mn}$ and $\mathrm{Ni}$ [27]. Tunneling spectroscopy was used to determine the electronic structure of the chains revealing no resonances below $1 \mathrm{eV}$ (see Supplemental Material [28]). Using Sp-STM with in-plane magnetized $\mathrm{Fe}$ coated tips, we investigated the magnetic (a)

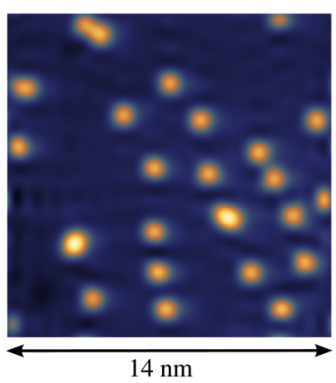

(b)

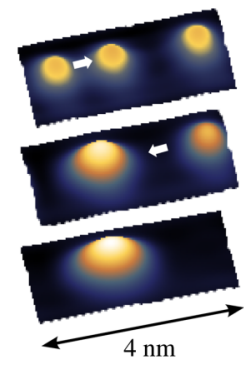

FIG. 2 (color online). STM images of Mn atoms on $\mathrm{Ni}(110)$. (a) Sample with 0.02 ML Mn/Ni(110) deposited at 4.2 K showing mainly isolated atoms. (b) Formation of a linear Mn trimer by atomic manipulation.

structure of Mn chains on the atomic scale. For this, we chose a bias voltage of $350 \mathrm{mV}$ as it revealed a strong spin contrast, as shown later, and is far away from the experimentally observed standing waves within the chains (see Supplemental Material [28]). In general, the chains appear darker than the Ni substrate in $d I / d U$ images at this bias voltage, as their local density of states is lower. Thus, we focus only on the contrast within the Mn chains as only this can be related to a magnetic signal. For trimers, the measurement revealed a strong spin contrast along the chain [see center panel in Fig. 3(b)]. Similarly, the line section along the trimer axis displays two minima at the edge atoms and a maximum in the center. This is in full agreement with predictions of antiferromagnetic odd-numbered chains displaying a simple collinear spin structure (see
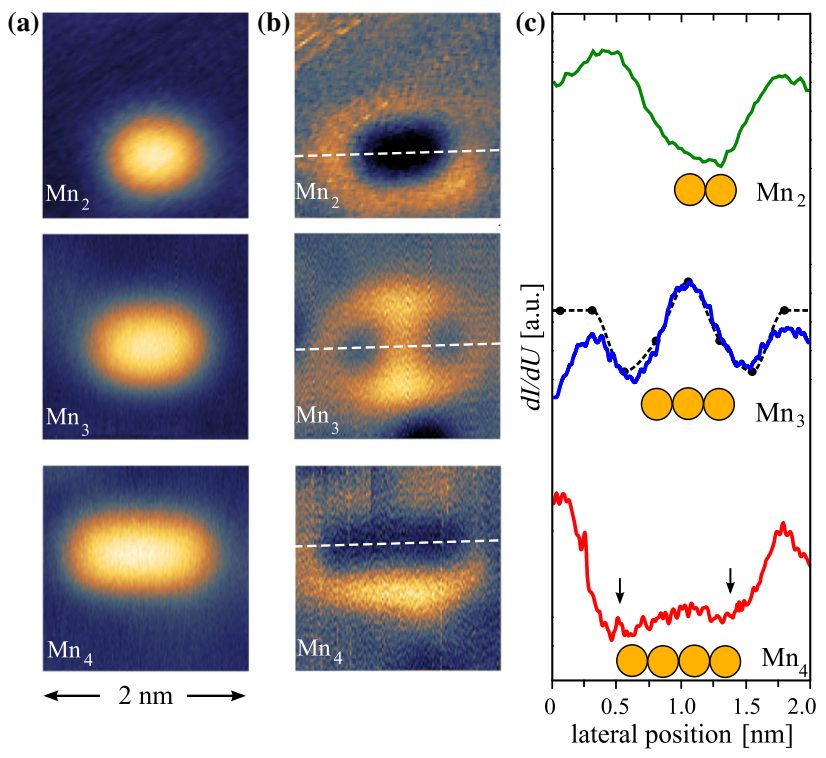

FIG. 3 (color online). Sp-STM investigation of linear Mn clusters on $\mathrm{Ni}(110)$. (a) Topographic images of a Mn dimer, trimer and tetramer (from top to bottom). (b) Corresponding Sp-STM results all obtained with the same Fe-coated tip at $350 \mathrm{mV}$ and $6 \mathrm{nA}$. (c) Linescans along the dashed lines in the corresponding images (b) including the calculated local spin density of states of the trimer (dashed line in the center panel). 
Fig. 1). Note, however, that the distance between the minima appears slightly larger than the geometrical distance between the $\mathrm{Mn}$ atoms assuming a distance of $0.249 \mathrm{~nm}$ corresponding to the distance of the adsorption sites on the $\mathrm{Ni}(110)$ surface. This is mainly caused by the fact that STM probes the electronic states about $0.5-1 \mathrm{~nm}$ out in the vacuum, i.e., the evanescent states. This leads to sizable smearing of atomically sized objects and to an increase in apparent lengths and widths of the chains when compared to a hard sphere model. For an antiferromagnetic trimer, the two outer atoms appear as dips and the central one as a peak and upon measuring the evanescent states, the positions of minima shift outward due to smearing. This experimental finding can easily be reproduced by calculating the local spin density along the trimer plotted as a dashed line in the center panel in Fig. 3(c).

The dimer [upper panels in Figs. 3(b) and 3(c)] does not show a contrast in agreement with the predictions. In the calculated magnetic configuration, the projection of the individual Mn magnetic moments on the substrate magnetization are identical. The tetramer also does not display a strong contrast [see lower panels in Figs. 3(b) and 3(c)]. On closer inspection, the line section shows a small depression of the $d I / d U$ signal at the edge atoms of the chain [arrows in Fig. 3(c)]. This is expected for the tetramer, as the edge atoms align more to the Ni substrate moments. Thus, all chains show the expected projections of the magnetic moment along the substrate magnetization.

Dimers and tetramers were suggested to have a noncollinear magnetic structure and, as a consequence, they should display a more complex spin contrast when using a tip magnetized perpendicular to the $\mathrm{Ni}$ moments. In experiments with different $\mathrm{Mn}$ tips of random magnetic orientation, $\mathrm{Fe}$ tips with arbitrary in-plane orientation, or Co tips with out-of-plane orientation, we, however, never observed such a contrast. This rather suggests that the expectation value of the Mn moments perpendicular to the magnetization direction of the substrate does not vary along the even-numbered chains under the experimental conditions. Possibly, spin fluctuations too fast to be observed with STM are present, such that the noncollinear components of the magnetization of the Mn chain are averaged to zero within the typical observation time (10 ms per pixel). Such spin fluctuations might be caused by thermal excitation or by inelastic spin scattering with the electrons of the tunneling current.

We, however, argue in the following that when treating the energy of the spin configuration within classical equations, we do not predict a classical ground state but a nonclassical state due to zero-point motion even at zero temperature [5]. The complexity of the magnetic interactions in noncollinear structures with spin-orbit interactions hinders the use of $a b$ initio methods beyond DFT, e.g., a time-dependent procedure (see for example Refs. [29,30]). Also a model Hamiltonian of a quantum Heisenberg system with localized spins and exact diagonalization [21] describing effects like magnetization tunneling [31] cannot be used, as it neglects the itinerant nature of the system. Instead, we take a new pathway to describe even-numbered chains following ideas of Leggett et al. [32] in the framework of the "spin-boson" problem. By integrating out the electronic degrees of freedom described within DFT, we compute the interatomic exchange interaction and the intra-atomic spinorbit interaction. The parameters extracted from DFT are then fed into classical equations of motion. The resulting equations are then treated in the limit of quantum mechanics to investigate the spin-dynamics of the system.

The equation of motion [33] for a magnetic atom without damping is given by

$$
\frac{\partial \mathbf{M}}{\partial t}=-\gamma \mathbf{M} \times \mathbf{H}_{\mathrm{eff}},
$$

where $\mathbf{H}_{\text {eff }}$ is the effective magnetic field acting on the magnetic moment $\mathbf{M}$, and $\gamma$ is the gyromagnetic ratio. $\mathbf{H}_{\text {eff }}$ can be determined from the total energy according to

$$
\mathbf{H}_{\mathrm{eff}}=\frac{-\nabla_{\mathbf{M}} E}{\gamma},
$$

where $E$ is the total energy corresponding to a Heisenberg Hamiltonian, which includes magnetic anisotropy and in which the magnetic exchange energies between first neighbors are considered. For the Mn dimer we find

$$
\begin{aligned}
E(\theta, \phi)= & -J_{1} \cos (2 \theta)-2 J_{2} \cos (\theta) \\
& +2 K \sin ^{2}(\theta) \cos ^{2}(\phi) .
\end{aligned}
$$

Here, $\theta$ is the polar angle of Mn magnetization to the magnetization direction of the Ni substrate, and $\phi$ is the azimuthal angle of the magnetization direction of the $\mathrm{Mn}$ atoms with respect to the easy axis. The Ni moments were treated as rigid. $J_{1}$ and $J_{2}$ are the exchange constants for Mn-Mn and Ni-Mn exchange, respectively. $K$ is the uniaxial anisotropy per adatom of the Mn dimer for rotation around the Ni magnetization direction. The equation describes the coupled dynamics of $\theta$ and $\phi$ in a harmonic potential for small deviations from the ground state with $\phi_{0}=0^{\circ}, 180^{\circ}$.

From our $a b$ initio calculations, we determine $J_{1}=$ $-221.4 \mathrm{meV}, \quad J_{2}=116.8 \mathrm{meV}$, and $K=-0.3 \mathrm{meV}$, which result in $\theta_{0}=75^{\circ}$ and an easy direction of the Mn moments along the surface normal. The energy barrier to coherently and adiabatically rotate the moments of the $\mathrm{Mn}$ atoms is proportional to the anisotropy barrier $K$ multiplied by $2 \sin ^{2}\left(\theta_{0}\right)=0.56 \mathrm{meV}$. When solving the coupled equation of motion in $\theta$ and $\phi$ around the ground state, we obtain an eigenfrequency $\omega$ for the precession of the magnetic moments. This precession involves both $\phi$ and $\theta$ (see, e.g., Ref. [34]). It corresponds to a periodic oscillation of the two variables in a local well of the potential. It can be treated to lowest order as a harmonic oscillator. This frequency defines thus a zero-point fluctuation energy $E_{0}$, 


$$
E_{0}=\frac{1}{2} \hbar \omega=\frac{g \mu_{B}}{2 M} \sqrt{-4 K \cos \left(2 \phi_{0}\right)\left[4 J_{1} \cos \left(2 \theta_{0}\right)+2 J_{2} \cos \left(\theta_{0}\right)+4 K \cos \left(2 \theta_{0}\right) \cos ^{2}\left(\phi_{0}\right)\right]-\left[4 K \cos \left(\theta_{0}\right) \sin \left(2 \phi_{0}\right)\right]^{2}},
$$

where $g \approx 2$ is the $g$ factor and $\mu_{B}$ the Bohr magneton. Surprisingly, the exchange interactions also enter the zero-point fluctuation energy, leading to $E_{0}$ of the order of $8.9 \mathrm{meV}$. Thus, in the ground state, the magnetic moments have a much larger fluctuation energy than the anisotropy barrier, and the system can overcome the barrier.

Similar equations for the tetramer also reveal that the zero-point motion is large enough to overcome the anisotropy barrier (see Supplemental Material [28]). In the framework of the "spin-boson" problem, this case represents the situation where the matrix element for tunneling between the two localized states (mediated by the exchange interaction) is larger than the barrier (mediated by the spin-orbit interaction). In this pathological case of the "spin-boson" problem, the states do not localize in one or the other classical state even at $T=0 \mathrm{~K}$ [32]. It is crucial and very instructive to analyze the previous equation: The barrier height and $E_{0}$ depend on both the anisotropy and the exchange energies. While the maximal value of the barrier height is limited by the anisotropy, the zeropoint energy strongly depends on the exchange constants. One notices that for typical values of the exchange being much larger than the anisotropy, the zero-point energy is also much larger than the anisotropy. By decreasing $J_{1}$ and omitting $J_{2}$ (paramagnetic substrate), the zero-point energy decreases quickly. Thus at the limit of weak interactions between the adatoms and a finite barrier due to anisotropy, the system exhibits a localized, i.e., Néel, ground state [35].

In conclusion, we have shown that although there is a finite magnetic anisotropy energy acting as a potential barrier between the degenerate DFT ground states in even-numbered chains, a zero-point energy provides a mean for fluctuations between the two degenerate states. The latter one is found to be surprisingly large and should be considered when describing antiferromagnetic nanoobjects. Just by adding or removing one atom of the chain - changing parity - the system changes its magnetic behavior completely and behaves classically. This is due to the net spin of the chain coupling to the macroscopic magnetization of the $\mathrm{Ni}$ substrate. We believe that our findings can show a path to create magnetically stable antiferromagnetic structures, i.e., to raise the blocking temperature. This intriguing result is obtained without requiring a quantum Heisenberg model but by treating the equation of motion for the magnetic moments as a quantum equation. In this respect, we believe that this approach is general and could lead to a better understanding of the dynamics of small spin systems.

S. L. acknowledges discussions with P. H. Dederichs and the support of the HGF-YIG Programme No. VH-NG-717
(Functional nanoscale structure and probe simulation laboratory-Funsilab). W. W. acknowledges discussions with J. Schmalian and funding by the Deutsche Forschungsgemeinschaft (DFG Grant No. WU 349/4-1).

[1] M. N. Leuenberger and D. Loss, Nature (London) 410, 789 (2001).

[2] L. Néel, Ann. de Phys. 5, 232 (1936).

[3] L. Balents, Nature (London) 464, 199 (2010).

[4] C. Lacroix, J. Phys. Soc. Jpn. 79, 011008 (2010).

[5] P. W. Anderson, Phys. Rev. 102, 1008 (1956).

[6] G. H. Wannier, Phys. Rev. 79, 357 (1950).

[7] W. Wulfhekel and J. Kirschner, Annu. Rev. Mater. Res. 37, 69 (2007).

[8] C. L. Gao, W. Wulfhekel, and J. Kirschner, Phys. Rev. Lett. 101, 267205 (2008).

[9] M. Waśniowska, S. Schröder, P. Ferriani, and S. Heinze, Phys. Rev. B 82, 012402 (2010).

[10] S. Lounis, P. H. Dederichs, and S. Blügel, Phys. Rev. Lett. 101, 107204 (2008).

[11] P. Wahl, P. Simon, L. Diekhöner, V.S. Stepanyuk, P. Bruno, M. A. Schneider, and K. Kern, Phys. Rev. Lett. 98, 056601 (2007).

[12] D. M. Eigler and E. K. Schweizer, Nature (London) 344, 524 (1990).

[13] N. Papanikolaou, R. Zeller, and P. H. Dederichs, J. Phys. Condens. Matter 14, 2799 (2002).

[14] H. Ebert and R. Zeller, http://olymp.cup.uni-muenchen.de/ ak/ebert/SPR-TB-KKR.

[15] S. Lounis, P. Mavropoulos, P. H. Dederichs, and S. Blügel, Phys. Rev. B 72, 224437 (2005).

[16] A. Liechtenstein, J. Magn. Magn. Mater. 67, 65 (1987).

[17] The total energy also remains unchanged in the case of noncollinear substrate magnetization. When rotating the $\mathrm{Mn}$ moments, and with it the noncollinear part of the $\mathrm{Ni}$ magnetization, around the average $\mathrm{Ni}$ magnetization direction, the exchange energy does not alter.

[18] The anisotropy has been calculated from the change of the total energy upon coherent rotation of all moments around the average $\mathrm{Ni}$ magnetization direction and thus also includes a small contribution from the noncollinear components of the Ni substrate.

[19] S. Heinze, M. Bode, A. Kubetzka, O. Pietzsch, X. Nie, S. Blügel, and R. Wiesendanger, Science 288, 1805 (2000).

[20] C.L. Gao, A. Ernst, A. Winkelmann, J. Henk, W. Wulfhekel, P. Bruno, and J. Kirschner, Phys. Rev. Lett. 100, 237203 (2008).

[21] C. F. Hirjibehedin, C. P. Lutz, and A. J. Heinrich, Science 312, 1021 (2006).

[22] F. Meier, L. Zhou, J. Wiebe, and R. Wiesendanger, Science 320, 82 (2008).

[23] T. Balashov, T. Schuh, A. F. Takács, A. Ernst, S. Ostanin, J. Henk, I. Mertig, P. Bruno, T. Miyamachi, S. Suga et al., Phys. Rev. Lett. 102, 257203 (2009). 
[24] L. Zhou, J. Wiebe, S. Lounis, E. Vedmedenko, F. Meier, S. Blügel, P. H. Dederichs, and R. Wiesendanger, Nat. Phys. 6, 187 (2010).

[25] T. Miyamachi, T. Schuh, T. Balashov, S. Suga, and W. Wulfhekel, e-J. Surf. Sci. Nanotechnol. 9, 237 (2011).

[26] A. Khajetoorians, J. Wiebe, B. Chilian, S. Lounis, S. Blügel, and R. Wiesendanger, Nat. Phys. 8, 497 (2012).

[27] CRC Handbook of Chemistry and Physics, edited by D. R. Lide (CRC Press, London, 1995), 76th ed.

[28] See Supplemental Material at http://link.aps.org/ supplemental/10.1103/PhysRevLett.110.157206 for details of the electronic structure of the Mn chains, of the calculations, and of the classical analogon.
[29] S. Lounis, A. T. Costa, R. B. Muniz, and D. L. Mills, Phys. Rev. Lett. 105, 187205 (2010).

[30] S. Lounis, A. T. Costa, R. B. Muniz, and D. L. Mills, Phys. Rev. B 83, 035109 (2011).

[31] W. Wernsdorfer and R. Sessoli, Science 284, 133 (1999).

[32] A. Leggett, S. Chakravarty, A. Dorsey, M. Fisher, A. Garg, and W. Zwerger, Rev. Mod. Phys. 59, 1 (1987).

[33] T. Gilbert, IEEE Trans. Magn. 40, 3443 (2004).

[34] F. P. Valstyn, J. P. Hanton, and A. H. Morrish, Phys. Rev. 128, 2078 (1962).

[35] S. Loth, S. Baumann, C. P. Lutz, D. M. Eigler, and A. J. Heinrich, Science 335, 196 (2012). 